\title{
Quotients of Torus Endomorphisms and Lattès-Type Maps
}

\author{
Mario Bonk ${ }^{1}$ - Daniel Meyer ${ }^{2}$
}

Received: 30 January 2020 / Revised: 9 July 2020 / Accepted: 6 August 2020 /

Published online: 14 September 2020

(c) The Author(s) 2020

\begin{abstract}
We show that if an expanding Thurston map is the quotient of a torus endomorphism, then it has a parabolic orbifold and is a Lattès-type map.

Keywords Thurston maps · Lattès maps, Lattès-type maps · Quotients of torus endomorphisms · Parabolic orbifold
\end{abstract}

\section{Contents}

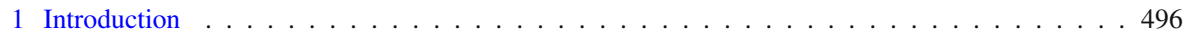

2 Background . . . . . . . . . . . . . . . . . . . . . . . 499

2.1 Branched Covering Maps . . . . . . . . . . . . . . . . . . . . . . . . . . . . . 499

2.2 Thurston Maps . . . . . . . . . . . . . . . . . . . . . . . . . . . . . . 500

2.3 Expansion . . . . . . . . . . . . . . . . . . . . . . . . 501

2.4 Thurston Equivalence . . . . . . . . . . . . . . . . . . . . . . . . . . 501

2.5 The Orbifold Associated with a Thurston Map . . . . . . . . . . . . . . . . . . . . . 502

2.6 Parabolic Orbifolds . . . . . . . . . . . . . . . . . . . . . . . 502

2.7 Lattès Maps . . . . . . . . . . . . . . . . . . . . . . . . . . . . . . . . . 503

2.8 Quotients of Torus Endomorphisms . . . . . . . . . . . . . . . . . . . . . . 504

2.9 Lattès-Type Maps . . . . . . . . . . . . . . . . . . . . . . . . . . . 505

2.10 Lattices and Tori . . . . . . . . . . . . . . . . . . . . . . . . . 507

2.11 Lifts by Branched Covering Maps _ . . . . . . . . . . . . . . . . . . . . . 508

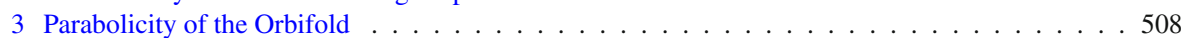

3.1 Expansion Properties . . . . . . . . . . . . . . . . . . . . . . . 510

3.2 Transitive Action on Fibers . . . . . . . . . . . . . . . . . . . . . . . . 514

4 From Parabolic Orbifolds to Lattès-Type Maps . . . . . . . . . . . . . . . . . . . . . . . 518

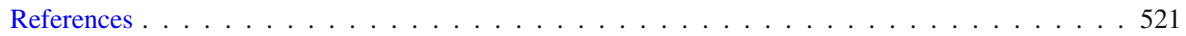

Mario Bonk was partially supported by NSF grant DMS-1808856.

$凶$ Daniel Meyer

dmeyermail@gmail.com

Mario Bonk

mbonk@math.ucla.edu

1 Department of Mathematics, University of California, Los Angeles, CA 90095, USA

2 Department of Mathematical Sciences, University of Liverpool, Liverpool L69 7ZL, UK 


\section{Introduction}

The main purpose of this paper is to present an open problem about Thurston maps that has mystified the authors while writing [1]. The general underlying question is which properties of a Thurston map are of a purely topological nature, or whether more geometric or even analytic structure is required to characterize a property. Our problem is closely related to certain classes of maps, namely Lattès and Lattès-type maps. We start with recalling some background about these classes.

Lattès maps are rational maps on the Riemann sphere $\widehat{\mathbb{C}}=\mathbb{C} \cup\{\infty\}$ that are given as quotient maps of holomorphic torus endomorphisms. More precisely, a map $f: \widehat{\mathbb{C}} \rightarrow \widehat{\mathbb{C}}$ is a Lattès maps if and only if there exist a (non-homeomorphic and non-constant) holomorphic map $\bar{A}: \mathbb{T} \rightarrow \mathbb{T}$ on a complex torus $\mathbb{T}$ and a non-constant holomorphic map $\bar{\Theta}: \mathbb{T} \rightarrow \widehat{\mathbb{C}}$, such that we have the following commutative diagram:

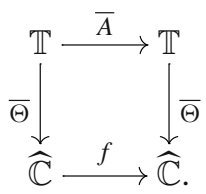

Here, a complex torus $\mathbb{T}$ is a Riemann surface whose underlying 2-manifold is a 2-dimensional torus.

It is then not hard to see that $f$ is a holomorphic map and, hence, a rational map on $\widehat{\mathbb{C}}$. Moreover, one can show (see Theorem 2.5) that every Lattès map $f$ is actually a postcritically finite rational map with a parabolic orbifold (we explain this terminology in Sect. 2). Verifying that a map $f$ as in (1.1) has, indeed, a parabolic orbifold is the difficult part in the proof of this statement. The argument uses the holomorphicity of $f$ in an essential way (see [1, p. 64] and [4]).

Thurston raised the question when a map that behaves as a rational map in a certain topological way is actually "equivalent" to a rational map (see [3] and [1] for a systematic study of this point of view). In view of this, it is natural to consider topological analogs of maps as in (1.1). This means that we consider maps $f: S^{2} \rightarrow S^{2}$ with the property that there exists a torus endomorphism $\bar{A}: T^{2} \rightarrow T^{2}$ (i.e., an unbranched covering map) with topological degree $\operatorname{deg}(\bar{A}) \geq 2$, as well as a branched covering map $\bar{\Theta}: T^{2} \rightarrow S^{2}$, such that we have the following commutative diagram:

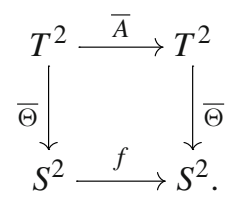

Here, $S^{2}$ is a topological 2-sphere, and $T^{2}$ is a topological 2-torus. We use notation different from (1.1) to indicate that these are topological objects and not Riemann surfaces, meaning that the surfaces are not equipped with a conformal structure. 
If a map $f$ arises as in (1.2), then we call $f$ a quotient of a torus endomorphism (see Definition 2.6 for a precise statement). One can show that such a map $f$ is actually a Thurston map, i.e., a non-homeomorphic branched covering map with a finite set of postcritical points (see Lemma 2.7). One should expect that these maps are closely related to Lattès maps. In particular, one expects a positive answer to the following question.

\section{Problem 1.1 Does every quotient of a torus endomorphism have a parabolic orbifold?}

We have repeatedly tried to tackle this problem and also consulted with various experts, but a convincing argument for a positive answer is elusive at this point. Accordingly, it seems appropriate to present a partial answer and some facts related to Problem 1.1. This is the main purpose of this paper.

To formulate our result, we first have to define Lattès-type maps. As this involves somewhat technical terminology, we will introduce these maps in an informal way for now, but will give a precise definition later in Sect. 2 (see Definition 2.8).

As a starting point, one notices (see the discussion at the beginning of Sect. 3) that by a lifting argument for each map $f: S^{2} \rightarrow S^{2}$ as in (1.2), one has a commutative diagram of the form:

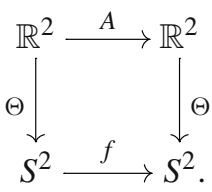

Here, $\Theta: \mathbb{R}^{2} \rightarrow S^{2}$ is a branched covering map and $A: \mathbb{R}^{2} \rightarrow \mathbb{R}^{2}$ is an orientationpreserving homeomorphism with a suitable equivariance property with respect to the group $G$ of deck transformations of $\Theta$.

In the special situation of (1.3) when $A$ is a (real) affine map on $\mathbb{R}^{2}$ and $G$ is a crystallographic group, one calls $f$ a Lattès-type map (see Definition 2.8). One can show that each Lattès map is also a Lattès-type map. This immediately follows from the characterization of Lattès maps as in condition (ii) of Theorem 2.5.

Moreover, each Lattès-type map is a quotient of a torus endomorphism with a parabolic orbifold (see Proposition 2.9). In general, the converse implication is not true, but our main result provides such a converse under the assumption that the Thurston map $f$ is expanding (see (2.3) for the precise definition).

Theorem 1.2 Let $f: S^{2} \rightarrow S^{2}$ be an expanding Thurston map. Then, the following conditions are equivalent:

(i) $f$ is the quotient of a torus endomorphism.

(ii) $f$ has a parabolic orbifold.

(iii) $f$ is a Lattès-type map.

Since every quotient $f$ of a torus endomorphism is actually a Thurston map, the previous statement gives an answer to Problem 1.1 if $f$ is expanding.

As we already pointed out, the implication (iii) $\Rightarrow$ (i) is known (by Proposition 2.9). The most difficult part in the proof of Theorem 1.2 is to establish the implication 
(i) $\Rightarrow$ (ii). Here, we cannot rely on holomorphicity as in the proof of the parabolicity of the orbifold of Lattès maps as defined in (1.1). Instead, we will use a dynamical argument based on the expansion properties of $f$ and its associated maps (see the considerations in Sect. 3 which lead to Proposition 3.7).

The proof of the implication (ii) $\Rightarrow$ (iii) relies on the fact that an expanding Thurston map $f$ with parabolic orbifold cannot have periodic critical points, which in turn implies that $f$ is Thurston equivalent to a Lattès-type map $g$ (see Proposition 2.10). We will show that $g$ is expanding (see the proof of Proposition 4.2). A standard result (Theorem 2.4) then implies that $f$ and $g$ are, in fact, topologically conjugate. We can then conclude that $f$ itself is a Lattès-type map (see Lemma 4.1).

One may ask to what extent some of these implications are true without the assumption that the Thurston map $f$ is expanding. For (i) $\Rightarrow($ ii), this leads to the open Problem 1.1. The implication (iii) $\Rightarrow$ (i) is still true without expansion (see Proposition 2.9). The relation between (ii) and (iii) is covered by the following statement: Let $f$ be a Thurston map. Then, $f$ is Thurston equivalent to a Lattès-type map if and only if $f$ has a parabolic orbifold and no periodic critical points (see Proposition 2.10). Note that Thurston maps with parabolic orbifolds and periodic critical points are also easy to classify up to Thurston equivalence: essentially, these are power maps $z \mapsto z^{n}$ and Chebyshev polynomials (see [1, Chapter 7]).

The paper is organized as follows. We review all the relevant background and preliminaries in Sect. 2. The proof of the implications (i) $\Rightarrow$ (ii) and (ii) $\Rightarrow$ (iii) in Theorem 1.2 are then given in Sects. 3 and 4. We wrap up the proof of Theorem 1.2 at the end of Sect. 4.

Notation When an object $A$ is defined to be another object $B$, we write $A:=B$ for emphasis.

We denote by $\mathbb{N}=\{1,2, \ldots\}$ the set of natural numbers and by $\mathbb{N}_{0}=\{0,1,2, \ldots\}$ the set of natural numbers including 0 . The sets of integers, real numbers, and complex numbers are denoted by $\mathbb{Z}, \mathbb{R}$, and $\mathbb{C}$, respectively. We let $\widehat{\mathbb{C}}:=\mathbb{C} \cup\{\infty\}$ be the Riemann sphere. We also consider $\widehat{\mathbb{N}}:=\mathbb{N} \cup\{\infty\}$. If $A \subset \widehat{\mathbb{N}}$, then $\operatorname{lcm}(A) \in \widehat{\mathbb{N}}$ denotes the least common multiple of the numbers in $A$.

When we consider two objects $A$ and $B$, and there is a natural identification between them that is clear from the context, we write $A \cong B$. For example, $\mathbb{R}^{2} \cong \mathbb{C}$ if we identify a point $(x, y) \in \mathbb{R}^{2}$ with $x+y \boldsymbol{i} \in \mathbb{C}$, where $\boldsymbol{i}$ is the imaginary unit.

The cardinality of a set $X$ is denoted by $\# X$ and the identity map on $X$ by id ${ }_{X}$. If $x_{n} \in X$ for $n \in \mathbb{N}$ are points in $X$, we denote the sequence of these points by $\left\{x_{n}\right\}_{n \in \mathbb{N}}$, or just by $\left\{x_{n}\right\}$ if the index set $\mathbb{N}$ is understood.

If $f: X \rightarrow X$ is a map and $n \in \mathbb{N}$, then:

$$
f^{n}:=\underbrace{f \circ \cdots \circ f}_{n \text { factors }}
$$

is the $n$th iterate of $f$. We set $f^{0}:=\operatorname{id}_{X}$ for convenience.

Let $f: X \rightarrow Y$ be a map between sets $X$ and $Y$. If $U \subset X$, then $f \mid U$ stands for the restriction of $f$ to $U$. If $A \subset Y$, then $f^{-1}(A):=\{x \in X: f(x) \in A\}$ is the 
preimage of $A$ in $X$. Similarly, $f^{-1}(y):=\{x \in X: f(x)=y\}$ is the preimage of a point $y \in Y$.

If $f: X \rightarrow X$ is a map, then preimages of a set $A \subset X$ or a point $p \in X$ under the $n$th iterate $f^{n}$ are denoted by $f^{-n}(A):=\left\{x \in X: f^{n}(x) \in A\right\}$ and $f^{-n}(p):=\left\{x \in X: f^{n}(x)=p\right\}$, respectively.

Let $(X, d)$ be a metric space, and $M \subset X$. Then, we denote by:

$$
\operatorname{diam}_{d}(M):=\sup \{d(x, y): x, y \in M\}
$$

the diameter of $M$. We drop the subscript $d$ here if the metric $d$ is clear from the context.

\section{Background}

In this section, we state some relevant definitions and collect some facts for the convenience of the reader. More details on all of these topics can be found in [1].

\subsection{Branched Covering Maps}

We closely follow the presentation in [1, Section 2.1 and Section A.6]. A surface is a connected and oriented topological 2-manifold. A surface $X$ is a topological disk if it is homeomorphic to the unit disk $\mathbb{D}:=\{z \in \mathbb{C}:|z|<1\}$.

Let $X$ and $Y$ be surfaces, and $f: X \rightarrow Y$ be a continuous map. Then, $f$ is a branched covering map if, for each point $q \in Y$, there exists a topological disk $V \subset Y$ with $q \in V$ that is evenly covered by $f$ in the following sense: for some index set $I \neq \emptyset$, we can write $f^{-1}(V)$ as a disjoint union:

$$
f^{-1}(V)=\bigcup_{i \in I} U_{i}
$$

of open sets $U_{i} \subset X$, such that $U_{i}$ contains precisely one point $p_{i} \in f^{-1}(q)$. Moreover, we require that for each $i \in I$, there exists $d_{i} \in \mathbb{N}$, and orientation-preserving homeomorphisms $\varphi_{i}: U_{i} \rightarrow \mathbb{D}$ and $\psi_{i}: V \rightarrow \mathbb{D}$ with $\varphi_{i}\left(p_{i}\right)=0$ and $\psi_{i}(q)=0$, such that:

$$
\left(\psi_{i} \circ f \circ \varphi_{i}^{-1}\right)(z)=z^{d_{i}}
$$

for all $z \in \mathbb{D}$.

For given $f$, the number $d_{i}$ is uniquely determined by $p=p_{i}$ and called the local degree of $f$ at $p$, denoted by $\operatorname{deg}(f, p)$. Our definition allows different local degrees at points in the same fiber $f^{-1}(q)$.

Every branched covering map $f: X \rightarrow Y$ is surjective, open (images of open sets are open), and discrete (the preimage set of every point is discrete in $X$, i.e., it has no limit points in $X$ ). Every (locally orientation-preserving) covering map (see [1, Section A.5]) is also a branched covering map. 
A critical point of a branched covering map $f: X \rightarrow Y$ is a point $p \in X$ with $\operatorname{deg}(f, p) \geq 2$. A critical value is a point $q \in Y$, such that the fiber $f^{-1}(q)$ contains a critical point of $f$. The set of critical points of $f$ is discrete in $X$ and the set of critical values of $f$ is discrete in $Y$. If $f: X \rightarrow Y$ is a branched covering map, then $f$ is an orientation-preserving local homeomorphism near each point $p \in X$ that is not a critical point of $f$.

If $X$ is a compact surface and $f: X \rightarrow X$ is a branched covering map, then we denote by $\operatorname{deg}(f) \in \mathbb{N}$ the topological degree of $f$ (see [1, Section 2.1 and A.4] for the precise definition and more discussion).

The following statement is useful if one has to deal with compositions of branched covering maps (see [1, Lemma A.16]).

Lemma 2.1 (Compositions of branched covering maps) Let $X, Y$, and $Z$ be surfaces, and $f: X \rightarrow Z, g: Y \rightarrow Z$, and $h: X \rightarrow Y$ be continuous maps, such that $f=g \circ h$.

(i) If $g$ and $h$ are branched covering maps, and $Y$ and $Z$ are compact, then $f$ is also a branched covering map.

(ii) If $f$ and $g$ are branched covering maps, then $h$ is a branched covering map. Similarly, if $f$ and $h$ are branched covering maps, then $g$ is a branched covering map.

Let $X, Y$, and $Z$ be surfaces, and $h: X \rightarrow Y, g: Y \rightarrow Z$ be branched covering maps. If $g \circ h: X \rightarrow Z$ is also a branched covering map, then we have:

$$
\operatorname{deg}(g \circ h, x)=\operatorname{deg}(g, h(x)) \cdot \operatorname{deg}(h, x)
$$

for all $x \in X$. We will use this multiplicativity of local degrees throughout, usually without specific reference. For the proof, we refer to [1, Lemma A.17]. Note that there slightly stronger assumptions were used, but the proof for (2.2) remains valid without change.

\subsection{Thurston Maps}

Throughout this paper, $S^{2}$ denotes a topological 2-sphere. We assume that $S^{2}$ is equipped with a fixed orientation. To be able to use metric language, we also assume that $S^{2}$ carries a base metric that induces the given topology on $S^{2}$.

Let $f: S^{2} \rightarrow S^{2}$ be a branched covering map. We denote by:

$$
\operatorname{crit}(f):=\left\{p \in S^{2}: \operatorname{deg}(f, p) \geq 2\right\}
$$

its (finite) set of critical points and by

$$
\operatorname{post}(f)=\bigcup_{n \geq 1}\left\{f^{n}(c): c \in \operatorname{crit}(f)\right\}
$$

its set of postcritical points. One can show that $\operatorname{post}\left(f^{n}\right)=\operatorname{post}(f)$. If $\operatorname{post}(f)$ is a finite set and $\operatorname{deg}(f) \geq 2$, we call $f$ a Thurston map. We also say that $f$ is postcritically 
finite. If, in addition, $f$ is defined on $\widehat{\mathbb{C}}$ and holomorphic, then $f$ is a postcritically finite rational map and we call $f$ a rational Thurston map. A periodic point of $f$ is a point $p \in S^{2}$ with $f^{n}(p)=p$ for some $n \in \mathbb{N}$. For more details, see [1, Section 2.1].

\subsection{Expansion}

Let $f: S^{2} \rightarrow S^{2}$ be a Thurston map. We say that $f$ is expanding if, for some Jordan curve $\mathcal{C} \subset S^{2}$ with $\operatorname{post}(f) \subset \mathcal{C}$, we have:

$$
\lim _{n \rightarrow \infty} \operatorname{mesh}(f, n, \mathcal{C})=0
$$

Here, $\operatorname{mesh}(f, n, \mathcal{C})$ denotes the supremum of the diameters of components of $S^{2} \backslash f^{-n}(\mathcal{C})$. This condition is independent of the choice of the curve $\mathcal{C}$ and the base metric on $S^{2}$ (see [1, Section 6.1]).

If $f: \widehat{\mathbb{C}} \rightarrow \widehat{\mathbb{C}}$ is a rational Thurston map, then it is expanding if and only if $f$ has no periodic critical points. This is the case if and only if its Julia set is the whole sphere $\widehat{\mathbb{C}}$ (see [1, Proposition 2.3]).

Every expanding Thurston map $f: S^{2} \rightarrow S^{2}$ has an associated visual metric on $S^{2}$ that induces the given topology. The metric $\varrho$ has an associated expansion factor $\Lambda>1$. We refer to [1, Chapter 8] for precise definitions. We will only need one fact about visual metrics.

Lemma 2.2 Let $f: S^{2} \rightarrow S^{2}$ be an expanding Thurston map, and $\varrho$ be a visual metric for $f$ with expansion factor $\Lambda>1$. Then, there exist constants $\delta_{\varrho}>0$ and $C>0$, such that for each path $\alpha:[0,1] \rightarrow S^{2}$ with $\operatorname{diam}_{\varrho}(\alpha)<\delta_{\varrho}$, each $n \in \mathbb{N}$, and each path $\tilde{\alpha}:[0,1] \rightarrow S^{2}$ with $f^{n} \circ \widetilde{\alpha}=\alpha$, we have:

$$
\operatorname{diam}_{\varrho}(\widetilde{\alpha}) \leq C \Lambda^{-n}
$$

This follows from [1, Lemma 8.9] and the discussion after this lemma. In other words, if we lift a path with sufficiently small diameter under $f^{n}$, then the lifts shrink uniformly at an exponential rate as $n \rightarrow \infty$.

\subsection{Thurston Equivalence}

For Thurston maps, one often considers the following notion of equivalence (see [1, Section 2.4] for more explanations).

Definition 2.3 Let $f: S^{2} \rightarrow S^{2}$ and $\widehat{f}: \widehat{S}^{2} \rightarrow \widehat{S}^{2}$ be Thurston maps. Then, they are called Thurston equivalent if there exist homeomorphisms $h_{0}, h_{1}: S^{2} \rightarrow \widehat{S}^{2}$ that are isotopic relative to $\operatorname{post}(f)$ and satisfy $h_{0} \circ f=\widehat{f} \circ h_{1}$.

Here, $\widehat{S}^{2}$ is another topological 2-sphere.

Two maps $f: S^{2} \rightarrow S^{2}$ and $\widehat{f}: \widehat{S}^{2} \rightarrow \widehat{S}^{2}$ are called topologically conjugate if there exists a homeomorphism $h: S^{2} \rightarrow \widehat{S}^{2}$, such that $h \circ f=\widehat{f} \circ h$. It easily follows from the definitions that if two Thurston maps are topologically conjugate, then they are Thurston equivalent. The converse is true if the maps are expanding. 
Theorem 2.4 Let $f: S^{2} \rightarrow S^{2}$ and $\widehat{f}: \widehat{S}^{2} \rightarrow \widehat{S}^{2}$ be expanding Thurston maps that are Thurston equivalent. Then, they are topologically conjugate.

This is [1, Theorem 11.1].

\subsection{The Orbifold Associated with a Thurston Map}

We follow [1, Section 2.5]. Let $f: S^{2} \rightarrow S^{2}$ be a Thurston map. For a given $p \in S^{2}$, we set:

$$
\alpha_{f}(p)=\operatorname{lcm}\left\{\operatorname{deg}\left(f^{n}, q\right): q \in S^{2}, n \in \mathbb{N} \text {, and } f^{n}(q)=p\right\}
$$

Here, $\operatorname{lcm}(M) \in \widehat{\mathbb{N}}:=\mathbb{N} \cup\{\infty\}$ denotes the least common multiple of a set $M \subset \mathbb{N}$.

Note that $\alpha_{f}(p)=\infty$ is possible. This is true if and only if $p$ is contained in a critical cycle of $f$, i.e., $p$ is a fixed point and a critical point of $f^{n}$ for some $n \in \mathbb{N}$. It follows that $\alpha_{f}$ is finite (i.e., it does not take the value $\infty$ ) if and only if $f$ has no periodic critical points. Note that, in general, an expanding Thurston map may have periodic critical points (see [1, Example 12.21]), but not if it is rational (see [1, Proposition 2.3]). One can also show that $\alpha_{f}(p)=1$ if and only if $p \in S^{2} \backslash \operatorname{post}(f)$ (see [1, Proposition 2.9]).

The function $\alpha_{f}: S^{2} \rightarrow \widehat{\mathbb{N}}$ is called the ramification function of $f$ and $\mathcal{O}_{f}=$ $\left(S^{2}, \alpha_{f}\right)$ (i.e., the underlying 2 -sphere equipped with this ramification function) the orbifold associated with $f$. The Euler characteristic of $\mathcal{O}_{f}$ is defined as:

$$
\chi\left(\mathcal{O}_{f}\right)=2-\sum_{p \in S^{2}}\left(1-\frac{1}{\alpha_{f}(p)}\right) .
$$

For a Thurston map $f$, we always have $\chi\left(\mathcal{O}_{f}\right) \leq 0$ (see [1, Proposition 2.12]). We say that $f$ has a parabolic orbifold if $\chi\left(\mathcal{O}_{f}\right)=0$ and a hyperbolic orbifold if $\chi\left(\mathcal{O}_{f}\right)<0$.

\subsection{Parabolic Orbifolds}

To give a more precise classification of Thurston maps with parabolic orbifold, we consider the postcritical points $p_{1}, \ldots, p_{k}, k \in \mathbb{N}$, of a Thurston map $f$ labeled so that:

$$
\alpha_{f}\left(p_{1}\right) \leq \alpha_{f}\left(p_{2}\right) \leq \cdots \leq \alpha_{f}\left(p_{k}\right)
$$

The $k$-tuple $\left(\alpha_{f}\left(p_{1}\right), \ldots, \alpha_{f}\left(p_{k}\right)\right)$ is called the signature of $\mathcal{O}_{f}$.

The orbifold $\mathcal{O}_{f}$ associated with a Thurston map $f$ is parabolic, i.e., $\chi\left(\mathcal{O}_{f}\right)=0$, if and only if the signature of $\mathcal{O}_{f}$ is in the following list:

$$
(\infty, \infty),(2,2, \infty),(2,4,4),(2,3,6),(3,3,3),(2,2,2,2)
$$

(see [1, Proposition 2.14]). 
It follows from the definition of the ramification function $\alpha_{f}$ that $\operatorname{deg}(f, p) \alpha_{f}(p)$ divides $\alpha_{f}(f(p))$ for all $p \in S^{2}$ (see [1, Proposition 2.8 (ii)]). One can show that $\mathcal{O}_{f}$ is parabolic if and only if:

$$
\operatorname{deg}(f, p) \alpha_{f}(p)=\alpha_{f}(f(p))
$$

for all $p \in S^{2}$ (see [1, Proposition 2.14]).

\subsection{Lattès Maps}

We follow the presentation in [1, Chapter 3]. The definition of a Lattès map is based on the following fact (this is essentially well known; see [4] and [1, Theorem 3.1]).

Theorem 2.5 (Characterization of Lattès maps) Let $f: \widehat{\mathbb{C}} \rightarrow \widehat{\mathbb{C}}$ be a map. Then, the following conditions are equivalent:

(i) $f$ is a rational Thurston map that has a parabolic orbifold and no periodic critical points.

(ii) There exist a crystallographic group $G$, a $G$-equivariant holomorphic map $A: \mathbb{C} \rightarrow \mathbb{C}$ of the form $A(z)=\alpha z+\beta$, where $\alpha, \beta \in \mathbb{C},|\alpha|>1$, and $a$ holomorphic map $\Theta: \mathbb{C} \rightarrow \widehat{\mathbb{C}}$ induced by $G$, such that $f \circ \Theta=\Theta \circ A$.

(iii) There exist a complex torus $\mathbb{T}$, a holomorphic torus endomorphism $\bar{A}: \mathbb{T} \rightarrow \mathbb{T}$ with $\operatorname{deg}(\bar{A})>1$, and a non-constant holomorphic map $\bar{\Theta}: \mathbb{T} \rightarrow \widehat{\mathbb{C}}$, such that $f \circ \bar{\Theta}=\bar{\Theta} \circ \bar{A}$.

Here, a crystallographic group $G$ is a subgroup of the group of orientation-preserving isometries of $\mathbb{C}$ that acts properly discontinuously and cocompactly on $\mathbb{C}$. In particular, each element $g \in G$ is a map of the form $g: z \in \mathbb{C} \mapsto \alpha z+\beta$, where $\alpha, \beta \in \mathbb{C}$ with $|\alpha|=1$. Note that this definition of a crystallographic group $G$ is more restrictive than usual, because we require that all elements $g \in G$ preserve orientation on $\mathbb{C}$. These groups are completely classified (see [1, Theorem 3.7$]$ ).

A continuous map $\Theta: \mathbb{R}^{2} \rightarrow S^{2}$ is induced by a group $G$ of homeomorphisms on $\mathbb{R}^{2}$ if, for $u, v \in \mathbb{R}^{2}$, we have $\Theta(u)=\Theta(v)$ if and only if there exists $g \in G$, such that $v=g(u)$. The reason for this terminology is that under some additional assumptions (for example, when $\Theta$ is surjective and open), there exists a homeomorphism between $S^{2}$ and the quotient space $\mathbb{R}^{2} / G$, such that $\Theta$ corresponds to the quotient map $\mathbb{R}^{2} \rightarrow \mathbb{R}^{2} / G$ (see [1, Corollary A.23] for a precise statement along these lines). In the literature, such maps $\Theta$ are sometimes called strongly $G$-automorphic.

Finally, if $G$ is a group of homeomorphisms on $\mathbb{R}^{2}$ and $A: \mathbb{R}^{2} \rightarrow \mathbb{R}^{2}$ is a homeomorphism, then $A$ is called $G$-equivariant if

$$
A \circ g \circ A^{-1} \in G
$$

for each $g \in G$.

A map $f: \widehat{\mathbb{C}} \rightarrow \widehat{\mathbb{C}}$ on the Riemann sphere $\widehat{\mathbb{C}}$ is a Lattès map if one, hence each, of the conditions in Theorem 2.5 is satisfied. Such a map is always expanding (see 
[1, Proposition 2.3]). Note that condition (iii) in this theorem was how we introduced Lattès maps in the introduction.

In the following, for $h \in \mathbb{R}^{2} \cong \mathbb{C}$, we denote by $\tau_{h}: \mathbb{R}^{2} \rightarrow \mathbb{R}^{2}$ the translation defined as:

$$
\tau_{h}(u)=u+h, \quad \text { for } u \in \mathbb{R}^{2} .
$$

The subgroup of all translations in a crystallographic $G$ is denoted by $G_{\mathrm{tr}}$. One can show that for each crystallographic $G$, there exists a rank-2 lattice $\Gamma \subset \mathbb{R}^{2}$, such that $G_{\text {tr }}=\left\{\tau_{\gamma}: \gamma \in \Gamma\right\}$. In particular, $G_{\text {tr }}$ also acts cocompactly and properly discontinuously on $\mathbb{R}^{2}$. Moreover, the quotient space $\mathbb{T}=\mathbb{C} / G_{\mathrm{tr}} \cong \mathbb{R}^{2} / \Gamma$ is a torus carrying a natural complex structure, and it is hence a complex torus.

If $f$ is a Lattès map, then one can always find $A, \bar{A}, G, \bar{\Theta}$ as in Theorem 2.5 , such that we have the following commutative diagram (see $[1,(3.10)]$ ):

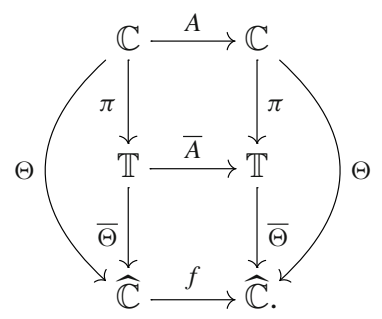

Here, $\pi: \mathbb{C} \rightarrow \mathbb{C} / G_{\mathrm{tr}}=\mathbb{T}$ is the quotient map which is the universal covering map of the torus $\mathbb{T}$. As we will see, a topological analog of (2.7) will be the starting point for the proof of Theorem 1.2.

\subsection{Quotients of Torus Endomorphisms}

We first record a precise definition for a quotient of a torus endomorphism. As before, we will denote by $T^{2}$ a 2-dimensional topological torus. We call a branched covering map $\bar{A}: T^{2} \rightarrow T^{2}$ a torus endomorphism. It easily follows from the RiemannHurwitz formula that $\bar{A}$ actually cannot have critical points, and so must be a (locally orientation-preserving) covering map. We can now give a precise definition of the most important concept in his paper.

Definition 2.6 (Quotients of torus endomorphisms) Let $f: S^{2} \rightarrow S^{2}$ be a map on a 2-sphere $S^{2}$ that satisfies the following condition: there exists a torus endomorphism $\bar{A}: T^{2} \rightarrow T^{2}$ with $\operatorname{deg}(\bar{A}) \geq 2$ and a branched covering map $\bar{\Theta}: T^{2} \rightarrow S^{2}$, such that $f \circ \bar{\Theta}=\bar{\Theta} \circ \bar{A}$. Then $f$ is called a quotient of a torus endomorphism.

In this case, we have a commutative diagram as in (1.2).

The following statement summarizes some facts about these maps (see [1, Lemmas 3.12 and 3.13]). 
Lemma 2.7 (Properties of quotients of torus endomorphisms) Let $f: S^{2} \rightarrow S^{2}$ be a quotient of a torus endomorphism, and $\bar{\Theta}: T^{2} \rightarrow S^{2}$ and $\bar{A}: T^{2} \rightarrow T^{2}$ with $\operatorname{deg}(\bar{A}) \geq 2$ be as in Definition 2.6. Then, the following statements are true:

(i) The map $f$ is a Thurston map without periodic critical points, and it satisfies $\operatorname{deg}(f)=\operatorname{deg}(\bar{A}) \geq 2$.

(ii) The set $\operatorname{post}(f)$ is equal to the set of critical values of $\bar{\Theta}$, that is:

$$
\operatorname{post}(f)=\bar{\Theta}(\operatorname{crit}(\bar{\Theta})) \text {. }
$$

(iii) $f$ has a parabolic orbifold if and only if

$$
\operatorname{deg}(\bar{\Theta}, x)=\operatorname{deg}(\bar{\Theta}, y)
$$

for all $x, y \in T^{2}$ with $\bar{\Theta}(x)=\bar{\Theta}(y)$.

This last parabolicity criterion will be important for us. The condition stipulates that the local degree $\operatorname{deg}(\bar{\Theta}, \cdot)$ is constant on the fiber $\bar{\Theta}^{-1}(p)$ for each $p \in S^{2}$.

\subsection{Lattès-Type Maps}

A map $L: \mathbb{R}^{2} \rightarrow \mathbb{R}^{2}$ is called $\mathbb{R}$-linear if:

$$
L(x+y)=L(x)+L(y) \text { and } L(\lambda x)=\lambda L(x)
$$

for all $x, y \in \mathbb{R}^{2}$ and $\lambda \in \mathbb{R}$. In other words, an $\mathbb{R}$-linear map $L: \mathbb{R}^{2} \rightarrow \mathbb{R}^{2}$ is a linear map on $\mathbb{R}^{2}$ considered as a vector space over $\mathbb{R}$. We write $\operatorname{det}(L) \in \mathbb{R}$ for the determinant of $L$.

A map $A: \mathbb{R}^{2} \rightarrow \mathbb{R}^{2}$ is called affine if it can be represented in the form:

$$
A(u)=L_{A}(u)+a, \quad u \in \mathbb{R}^{2},
$$

where $a \in \mathbb{R}^{2}$ and $L_{A}: \mathbb{R}^{2} \rightarrow \mathbb{R}^{2}$ is $\mathbb{R}$-linear. The map $L_{A}$ is uniquely determined by $A$ and called the linear part of $A$.

We can now give a precise definition of a Lattès-type map.

Definition 2.8 (Lattès-type maps) Let $f: S^{2} \rightarrow S^{2}$ be a map, such that there exist a crystallographic group $G$, an affine map $A: \mathbb{R}^{2} \rightarrow \mathbb{R}^{2}$ with $\operatorname{det}\left(L_{A}\right)>1$ that is $G$-equivariant, and a branched covering map $\Theta: \mathbb{R}^{2} \rightarrow S^{2}$ induced by $G$, such that $f \circ \Theta=\Theta \circ A$. Then, $f$ is called a Lattès-type map.

Note that then we have a commutative diagram as in (1.3). It follows from condition (ii) in Theorem 2.5 that every Lattès map is also of Lattès-type

Lattès-type maps are natural non-holomorphic analogs of Lattès maps. In this context, we usually write $\mathbb{R}^{2}$ instead of $\mathbb{C}$ for the plane, to emphasize that we do not rely on a complex structure. 
If $f: S^{2} \rightarrow S^{2}$ is a Lattès-type map, $G$ is a crystallographic group, and $\Theta: \mathbb{R}^{2} \rightarrow$ $S^{2}$ is induced by $G$ as in Definition 2.8 , then $G$ is necessarily of non-torus type, meaning that $G$ is not isomorphic to the rank-2 lattice $\mathbb{Z}^{2}$. This implies that there is a natural identification $\mathbb{R}^{2} / G \cong S^{2}$ of the quotient space $\mathbb{R}^{2} / G$ with the underlying 2sphere $S^{2}$. Under this identification, $\Theta$ corresponds to the quotient map $\mathbb{R}^{2} \rightarrow \mathbb{R}^{2} / G$ (see [1, Section 3.4]).

In the following, we summarize some facts about these maps. Note first, that if $f$ is a Lattès-type map, and $A$ is as in Definition 2.8 with its linear part $L_{A}$, then $\operatorname{det}\left(L_{A}\right)=\operatorname{deg}(f) \geq 2$ (see [1, Lemma 3.16]). This is the underlying reason for the requirement $\operatorname{det}\left(L_{A}\right)>1$ in Definition 2.8.

Some of the relations between Lattès-type maps, quotients of torus endomorphisms, and Thurston maps with parabolic orbifold are covered by the following two results.

Proposition 2.9 Every Lattès-type map $f: S^{2} \rightarrow S^{2}$ is a quotient of a torus endomorphism and, hence, a Thurston map. It has a parabolic orbifold and no periodic critical points.

This is [1, Proposition 3.5].

Proposition 2.10 A Thurston map $f: S^{2} \rightarrow S^{2}$ is Thurston equivalent to a Lattès-type map if and only if it has parabolic orbifold and no periodic critical points.

This is [1, Proposition 3.6]. Therefore, by (2.4), the signature of the orbifold of a Lattès-type map is in the list:

$$
(2,4,4),(2,3,6),(3,3,3),(2,2,2,2)
$$

Only the last signature leads to maps that are genuinely different from Lattès maps.

Proposition 2.11 A Lattès-type map $f: S^{2} \rightarrow S^{2}$ whose orbifold has signature $(2,4,4),(2,3,6)$, or $(3,3,3)$ is topologically conjugate to a Lattès map.

This is [1, Proposition 3.18]. A Lattès-type map with orbifold signature $(2,2,2,2)$ is in general not topologically conjugate (or Thurston equivalent) to a Lattès map (see [1, Theorem 3.22] and [3, Proposition 9.7]). These Lattès-type maps are examples of nearly Euclidean Thurston maps; see [2] for the definition.

A Lattès-type map is not necessarily expanding as a Thurston map (see [1, Example 6.15]). To record a criterion for this, we call an $\mathbb{R}$-linear map $L: \mathbb{R}^{2} \rightarrow \mathbb{R}^{2}$ expanding if $|\lambda|>1$ for each of the (possibly complex) roots $\lambda$ of the characteristic polynomial $P_{L}(z):=\operatorname{det}\left(L-z \operatorname{id}_{\mathbb{R}^{2}}\right)$ of $L$.

Proposition 2.12 Let $f: S^{2} \rightarrow S^{2}$ be a Lattès-type map and $L$ be the linear part of an affine map $A$ as in Definition 2.8. Then, $f$ is expanding (as a Thurston map) if and only if $L$ is expanding (as a linear map).

This is [1, Proposition 6.13]. 


\subsection{Lattices and Tori}

We quickly review some facts about lattices and tori (see [1, Section A.8] for more details).

A lattice $\Gamma \subset \mathbb{R}^{2}$ is a non-trivial discrete subgroup of $\mathbb{R}^{2}$ (considered as a group with vector addition). The rank of a lattice is the dimension of the subspace of $\mathbb{R}^{2}$ (considered as a real vector space) spanned by the elements in $\Gamma$. Here, we are only interested in rank-2 lattices $\Gamma$, i.e., lattices $\Gamma \subset \mathbb{R}^{2}$ that span $\mathbb{R}^{2}$.

If $\Gamma \subset \mathbb{R}^{2}$ is a rank-2 lattice, then the quotient space $\mathbb{R}^{2} / \Gamma$ (equipped with the quotient topology) is a 2-dimensional torus $T^{2}$, and the quotient map $\pi: \mathbb{R}^{2} \rightarrow T^{2}=$ $\mathbb{R}^{2} / \Gamma$ is a covering map. The lattice translations $\tau_{\gamma}, \gamma \in \Gamma$, are deck transformations of the quotient map $\pi$ and so $\pi=\pi \circ \tau_{\gamma}$ for $\gamma \in \Gamma$. Actually, every deck transformation of $\pi$ has this form (see [1, Lemma A.25 (i)]). Conversely, we may assume that any topological torus $T^{2}$ is given in the form $T^{2}=\mathbb{R}^{2} / \Gamma$ with some rank-2 lattice $\Gamma \subset \mathbb{R}^{2}$.

In the following lemma, we collect various statements that are used later.

Lemma 2.13 Let $\Gamma \subset \mathbb{R}^{2}$ be a rank-2 lattice, $T^{2}=\mathbb{R}^{2} / \Gamma$, and $\pi: \mathbb{R}^{2} \rightarrow T^{2}=\mathbb{R}^{2} / \Gamma$ be the quotient map.

(i) If $\bar{A}: T^{2} \rightarrow T^{2}$ is a torus endomorphism, then $\bar{A}$ can be lifted to a homeomorphism on $\mathbb{R}^{2}$, i.e., there exists a homeomorphism $A: \mathbb{R}^{2} \rightarrow \mathbb{R}^{2}$, such that $\bar{A} \circ \pi=\pi \circ A$. The homeomorphism A is orientation-preserving, and unique up to postcomposition with a translation $\tau_{\gamma}, \gamma \in \Gamma$.

(ii) If $\bar{A}: T^{2} \rightarrow T^{2}$ is a torus endomorphism, then there exists a unique invertible $\mathbb{R}$-linear map $L: \mathbb{R}^{2} \rightarrow \mathbb{R}^{2}$ with $L(\Gamma) \subset \Gamma$, such that for every lift $A$ as in $(i)$, we have:

$$
A \circ \tau_{\gamma} \circ A^{-1}=\tau_{L(\gamma)}=L \circ \tau_{\gamma} \circ L^{-1}
$$

for all $\gamma \in \Gamma$.

(iii) If $\bar{A}: T^{2} \rightarrow T^{2}$ is a torus endomorphism and $L$ is the map as in (ii), then $\operatorname{deg}(\bar{A})=$ $\operatorname{det}(L)$.

This is part of [1, Lemma A.25]. Note that there it was not explicitly stated that the linear map $L$ in (ii) is invertible. This was addressed in the proof though: one observes that the inclusion $L(\Gamma) \subset \Gamma$ and the relation $A \circ \tau_{\gamma} \circ A^{-1}=\tau_{L(\gamma)}$ for $\gamma \in \Gamma$ imply that the map $\gamma \in \Gamma \mapsto L(\gamma) \in \Gamma$ is injective. Therefore, $L: \Gamma \rightarrow \Gamma$ is an injective group homomorphism. Since $\Gamma$ is a rank-2 lattice, $L$ must be invertible as an $\mathbb{R}$-linear map on $\mathbb{R}^{2}$.

One can identify $\Gamma$ with the fundamental group of $T^{2}$. Then, the linear map $L$ is essentially the map on the fundamental group of $T^{2}$ induced by $\bar{A}$. For a careful explanation of this, see the discussion after [1, Lemma A.25]. 


\subsection{Lifts by Branched Covering Maps}

Since a Thurston map is a branched covering map, we need slight variants of the standard lifting theorems for unbranched covering maps. We list a useful uniqueness result (this is essentially [1, Lemma A.19 (i)]).

Lemma 2.14 Let $X, Y$, and $Z$ be surfaces and $f: X \rightarrow Y$ be a branched covering map. Suppose $g_{1}, g_{2}: Z \rightarrow X$ are continuous and discrete maps, such that $f \circ g_{1}=f \circ g_{2}$. If there exists a point $z_{0} \in Z$, such that $p:=g_{1}\left(z_{0}\right)=g_{2}\left(z_{0}\right)$ and $f(p) \in Y \backslash f(\operatorname{crit}(f))$, then $g_{1}=g_{2}$.

Note that $g_{1}$ and $g_{2}$ can be considered as lifts of the map $h:=f \circ g_{1}=f \circ g_{2}$ under $f$. Therefore, this is really a uniqueness statement for lifts under $f$.

The condition $y:=f(p) \in Y \backslash f(\operatorname{crit}(f))$ is the same as the requirement that $y$ is not a critical value of $f$, or equivalently, that the fiber $f^{-1}(y)$ contains no critical point of $f$. We will apply it in the case when $f: S^{2} \rightarrow S^{2}$ is a Thurston map. Then, this condition is satisfied if $p \in S^{2} \backslash f^{-1}(\operatorname{post}(f))$, because this implies that $f(p) \in S^{2} \backslash \operatorname{post}(f) \subset S^{2} \backslash f(\operatorname{crit}(f))$.

\section{Parabolicity of the Orbifold}

In this section, we will prove the implication (i) $\Rightarrow$ (ii) in Theorem 1.2. Throughout the section, we assume that $f: S^{2} \rightarrow S^{2}$ is a given quotient of a torus endomorphism that is expanding as a Thurston map (see Lemma 2.7 (i)). Then, there exists a torus $T^{2}$, and maps $\bar{A}: T^{2} \rightarrow T^{2}$ and $\bar{\Theta}: T^{2} \rightarrow S^{2}$ as in (1.2). We can identify $T^{2}$ with a quotient $\mathbb{R}^{2} / \Gamma$, where $\Gamma \subset \mathbb{R}^{2}$ is a rank-2 lattice, and we obtain a quotient map $\pi: \mathbb{R}^{2} \rightarrow T^{2} \cong \mathbb{R}^{2} / \Gamma$. The map $\bar{A}$ lifts to an orientation-preserving homeomorphism $A: \mathbb{R}^{2} \rightarrow \mathbb{R}^{2}$, such that $\pi \circ A=\bar{A} \circ \pi$ (this is standard and explicitly formulated in Lemma 2.13 (i)). We define $\Theta=\bar{\Theta} \circ \pi$. This is a branched covering map, since $\pi$ is a covering map and $\bar{\Theta}$ is a branched covering map (see Lemma 2.1 (i)). This leads to the following commutative diagram:

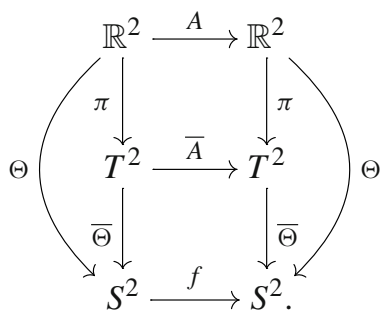

We denote by $G$ the group of all deck transformations of $\Theta$, i.e., the group of all homeomorphisms $g: \mathbb{R}^{2} \rightarrow \mathbb{R}^{2}$, such that $\Theta \circ g=\Theta$. Since $\Theta$ preserves orientation, the same is true for each homeomorphism $g \in G$.

Recall that $\tau_{h}$ for $h \in \mathbb{R}^{2}$ denotes the translation on $\mathbb{R}^{2}$ given by $\tau_{h}(u)=u+h$ for $u \in \mathbb{R}^{2}$. Then, $\pi \circ \tau_{\gamma}=\pi$ for $\gamma \in \Gamma$. This implies that all lattice translations $\tau_{\gamma}$, 
$\gamma \in \Gamma$, belong to $G$; indeed, for $\gamma \in \Gamma$, we have:

$$
\Theta \circ \tau_{\gamma}=\bar{\Theta} \circ \pi \circ \tau_{\gamma}=\bar{\Theta} \circ \pi=\Theta
$$

as required.

Our goal is to show that $f$ has a parabolic orbifold. To do so, we want to apply Lemma 2.7 (iii). Essentially, we have to show that the group $G$ of deck transformations acts transitively on each fiber of $\Theta$, i.e., on each of the sets $\Theta^{-1}(p), p \in S^{2}$. This means that we have to analyze some properties of the fixed map $\Theta$ in (3.1). Note that the diagram (3.1) remains valid with the same map $\Theta$, if we replace $A$ with $A^{\prime}=\tau_{\gamma} \circ A$ for any $\gamma \in \Gamma$. We can also replace $f, \bar{A}, A$ with iterates $f^{n}, \bar{A}^{n}, A^{n}$, respectively. We will make such replacements whenever this is convenient.

The map $A$ induces an invertible linear map $L: \mathbb{R}^{2} \rightarrow \mathbb{R}^{2}$, such that $L(\Gamma) \subset \Gamma$ and:

$$
A \circ \tau_{\gamma} \circ A^{-1}=\tau_{L(\gamma)}=L \circ \tau_{\gamma} \circ L^{-1}
$$

for all $\gamma \in \Gamma$ (see Lemma 2.13 (ii)). As we mentioned, this map $L$ can be viewed as the homomorphism induced by $\bar{A}$ on the fundamental group on $T^{2}$ (see the discussion after Lemma 2.13). If we use (3.2) repeatedly, then we see that:

$$
A^{n} \circ \tau_{\gamma} \circ A^{-n}=\tau_{L^{n}(\gamma)}=L^{n} \circ \tau_{\gamma} \circ L^{-n}
$$

for all $n \in \mathbb{N}$ and $\gamma \in \Gamma$.

If $x \in \mathbb{R}^{2}$ and $\gamma \in \Gamma$, then (3.2) implies that:

$$
\begin{aligned}
|A(x+\gamma)-L(x+\gamma)| & =\left|\left(A \circ \tau_{\gamma}\right)(x)-L(x)-L(\gamma)\right| \\
& =\left|\left(\tau_{L(\gamma)} \circ A\right)(x)-L(x)-L(\gamma)\right| \\
& =|A(x)-L(x)| .
\end{aligned}
$$

Since the lattice translations $\tau_{\gamma}, \gamma \in \Gamma$, act cocompactly on $\mathbb{R}^{2}$, it follows that there exists a constant $C_{0} \geq 0$, such that:

$$
|A(x)-L(x)| \leq C_{0} \text { for } x \in \mathbb{R}^{2} .
$$

Therefore, the maps $A$ and $L$ agree "coarsely" on large scales.

Before we go into more details, we outline the ensuing argument. Since $f$ is an expanding Thurston map, we first want to translate this expansion property of $f$ into expansion properties for the above maps $A$ and $L$. In particular, $L$ is an expanding linear map (Corollary 3.3). As we already mentioned, to prove that $f$ has a parabolic orbifold (see Proposition 3.7), we have to show that $G$ acts transitively on the fibers $\Theta^{-1}(p), p \in S^{2}$ (see Lemma 3.6). In [4], one can find related considerations for Lattès maps. There, the holomorphicity of the underlying maps is crucially used. Here, we will instead give a dynamical argument relying on the expansion property of $A$. We now proceed to establishing the details. 


\subsection{Expansion Properties}

We start with expansion properties of $A$. Actually, it is easier to formulate and prove contraction properties of $A^{-1}$. In the following, all metric notions on $\mathbb{R}^{2}$ refer to the Euclidean metric and all metric notions on $S^{2}$ to a fixed visual metric $\varrho$ for $f$ with expansion factor $\Lambda>1$.

Lemma 3.1 Let $\Theta: \mathbb{R}^{2} \rightarrow S^{2}$ be a map as (3.1). Then, the following statements are true:

(i) For each $\epsilon>0$, there exists $\delta>0$, such that for all $x, y \in \mathbb{R}^{2}$, we have:

$$
|x-y|<\delta \Rightarrow \varrho(\Theta(x),(\Theta(y))<\epsilon .
$$

(ii) For each $\epsilon>0$, there exists $\delta>0$, such that for each connected set $K \subset \mathbb{R}^{2}$, we have:

$$
\operatorname{diam}_{\varrho}(\Theta(K))<\delta \Rightarrow \operatorname{diam}(K)<\epsilon
$$

The statement and its proof are a small modification of the similar statement [1, Lemma 6.14].

Proof (i) The assertion is that $\Theta$ is uniformly continuous on $\mathbb{R}^{2}$. Essentially, this follows from the fact that the group $G$ of deck transformations of $\Theta$ contains the subgroup $G^{\prime}:=\left\{\tau_{\gamma}: \gamma \in \Gamma\right\}$ of all lattice translations and that this subgroup $G^{\prime}$ acts isometrically and cocompactly on $\mathbb{R}^{2}$.

In particular, we can find a compact fundamental domain $F \subset \mathbb{R}^{2}$ for the action of $G^{\prime}$ on $\mathbb{R}^{2}$. Now, suppose $x, y \in \mathbb{R}^{2}$ and $|x-y|$ is small. Then, there exists $g \in G^{\prime}$, such that $g(x) \in F$. If $|x-y|$ is small enough, as we may assume, then $g(x), g(y) \in U$, where $U$ is a fixed compact neighborhood of $F$. Since $\Theta$ is uniformly continuous on $U$, and $|g(x)-g(y)|=|x-y|$, it follows that:

$$
\varrho(\Theta(x), \Theta(y))=\varrho(\Theta(g(x)), \Theta(g(y))
$$

is small only depending on $|x-y|$. The uniform continuity of $\Theta$ follows.

(ii) We argue by contradiction and assume that the statement is false. Then, there exist connected sets $K_{n} \subset \mathbb{R}^{2}$, such that $\operatorname{diam}_{\varrho}\left(\Theta\left(K_{n}\right)\right) \rightarrow 0$ as $n \rightarrow \infty$, but $\operatorname{diam}\left(K_{n}\right) \geq \epsilon_{0}$ for $n \in \mathbb{N}$, where $\epsilon_{0}>0$.

We pick a point $x_{n} \in K_{n}$ for $n \in \mathbb{N}$. If we replace each set $K_{n}$ with its image $K_{n}^{\prime}=g_{n}\left(K_{n}\right)$ for suitable $g_{n} \in G^{\prime}$, where again $G^{\prime}:=\left\{\tau_{\gamma}: \gamma \in \Gamma\right\}$ and pass to a subsequence if necessary, then we may assume that the sequence $\left\{x_{n}\right\}$ converges, say $x_{n} \rightarrow x \in \mathbb{R}^{2}$ as $n \rightarrow \infty$. Note that $\operatorname{diam}\left(K_{n}^{\prime}\right)=\operatorname{diam}\left(K_{n}\right)$ and $\Theta\left(K_{n}^{\prime}\right)=\Theta\left(K_{n}\right)$.

Let $p:=\Theta(x)$. Since $\Theta: \mathbb{R}^{2} \rightarrow S^{2}$ is a branched covering map, the set $\Theta^{-1}(p)$ is discrete in $\mathbb{R}^{2}$ and consists of isolated points. In particular, $x \in \Theta^{-1}(p)$ is an isolated point of $\Theta^{-1}(p)$, and so, there exists a constant $m>0$, such that $|y-x| \geq m$ whenever $x, y \in \Theta^{-1}(p)$ and $x \neq y$. 
Pick a constant $c$ with $0<c<\min \left\{\epsilon_{0} / 2, m\right\}$. The set $K_{n}$ is connected, and has diameter $\operatorname{diam}\left(K_{n}\right) \geq \epsilon_{0}>2 c$. Hence, $K_{n}$ cannot be contained in the disk $\left\{z \in \mathbb{R}^{2}:\left|z-x_{n}\right|<c\right\}$, and so, it meets the circle $\left\{z \in \mathbb{R}^{2}:\left|z-x_{n}\right|=c\right\}$. It follows that there exists a point $y_{n} \in K_{n}$ with $\left|x_{n}-y_{n}\right|=c$. By passing to another subsequence if necessary, we may assume that the sequence $\left\{y_{n}\right\}$ converges, say $y_{n} \rightarrow y \in \mathbb{R}^{2}$ as $n \rightarrow \infty$. Then $|x-y|=c<m$. Note that $\Theta\left(x_{n}\right), \Theta\left(y_{n}\right) \in \Theta\left(K_{n}\right)$ for $n \in \mathbb{N}$, and $\operatorname{diam}_{\varrho}\left(\Theta\left(K_{n}\right)\right) \rightarrow 0$ as $n \rightarrow \infty$. Therefore:

$$
p=\Theta(x)=\lim _{n \rightarrow \infty} \Theta\left(x_{n}\right)=\lim _{n \rightarrow \infty} \Theta\left(y_{n}\right)=\Theta(y),
$$

and $x, y \in \Theta^{-1}(p)$. Since $|x-y|=c>0$, we have $x \neq y$. Then, $x$ and $y$ are two distinct points in $\Theta^{-1}(p)$ with $|x-y|=c<m$. This contradicts the choice of $m$, and the statement follows.

After this preparation, we now turn to the contraction properties of the map $A^{-1}$.

Lemma 3.2 Let the map $A: \mathbb{R}^{2} \rightarrow \mathbb{R}^{2}$ be as in (3.1). If $\epsilon_{1}, \epsilon_{2}>0$, then there exists $n_{0} \in \mathbb{N}$, such that:

$$
\left|A^{-n}(x)-A^{-n}(y)\right| \leq \epsilon_{1}|x-y|+\epsilon_{2}
$$

for all $x, y \in \mathbb{R}^{2}$ and $n \in \mathbb{N}$ with $n \geq n_{0}$.

The lemma essentially says that high iterates of $A^{-1}$ shrink distances that are not too small by an arbitrarily small factor. Conversely, by applying the statement to $x=A^{n}(u)$ and $y=A^{n}(v)$ for $u, v \in \mathbb{R}^{2}$, we see that sufficiently high iterates of $A$ expand distances that are not too small by an arbitrarily large factor.

Proof Let $\varrho$ be the visual metric for $f$ on $S^{2}$ that we fixed earlier and $\Lambda>1$ be the corresponding expansion factor. We also fix constants $\delta_{\varrho}>0$ and $C>0$ as in Lemma 2.2. Then, by Lemma 3.1 (i), we can find $\delta>0$ with the following property: if $\beta$ is a path in $\mathbb{R}^{2}$ with $\operatorname{diam}(\beta)<\delta$ and $\alpha=\Theta \circ \beta$, then $\operatorname{diam}_{\varrho}(\alpha)<\delta_{\varrho}$.

Now, suppose that $\beta$ is a path in $\mathbb{R}^{2}$ with $\operatorname{diam}(\beta)<\delta$. Then, the corresponding path $\alpha=\Theta \circ \beta$ satisfies $\operatorname{diam}_{\varrho}(\alpha)<\delta_{\varrho}$. For $n \in \mathbb{N}$, we also consider the paths $\widetilde{\beta}_{n}:=A^{-n} \circ \beta$ in $\mathbb{R}^{2}$, and $\widetilde{\alpha}_{n}:=\Theta \circ \widetilde{\beta}_{n}=\Theta \circ A^{-n} \circ \beta$ in $S^{2}$. From (3.1), we obtain:

$$
f^{n} \circ \widetilde{\alpha}_{n}=f^{n} \circ \Theta \circ A^{-n} \circ \beta=\Theta \circ A^{n} \circ A^{-n} \circ \beta=\Theta \circ \beta=\alpha .
$$

Therefore, $\widetilde{\alpha}_{n}$ is a lift of $\alpha$ under $f^{n}$. The relations between the paths are summarized in the following commutative diagram:

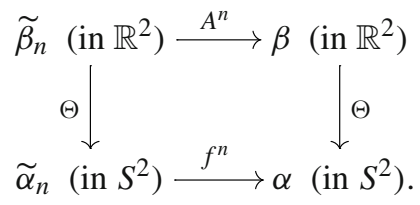


Since $\operatorname{diam}_{\varrho}(\alpha)<\delta_{\varrho}$, by the definition of $C$ and $\delta_{\varrho}$, we have:

$$
\operatorname{diam}_{\varrho}\left(\widetilde{\alpha}_{n}\right) \leq C \Lambda^{-n}
$$

Let $\epsilon_{1}, \epsilon_{2}>0$ be arbitrary, and $\tilde{\delta}:=\min \left\{\epsilon_{1} \delta, \epsilon_{2}\right\}>0$, where $\delta>0$ is chosen as above. Then, by Lemma 3.1, we can choose $\tilde{\delta}_{\varrho}>0$, such that $\operatorname{diam}\left(\widetilde{\beta}_{n}\right)<\tilde{\delta}$ whenever $\operatorname{diam}_{\varrho}\left(\widetilde{\alpha}_{n}\right)<\widetilde{\delta}_{\varrho}$ in $(3.5)$. Note that the constants $\delta, \delta_{\varrho}, \widetilde{\delta}_{\varrho}, \widetilde{\delta}_{\tilde{\delta}}$ serve to control the diameters of $\beta, \alpha, \widetilde{\alpha}_{n}, \widetilde{\beta}_{n}$, respectively.

Choose $n_{0} \in \mathbb{N}$, such that $C \Lambda^{-n}<\widetilde{\delta}_{\varrho}$ for $n \geq n_{0}$. Then:

$$
\operatorname{diam}\left(\widetilde{\beta}_{n}\right)<\widetilde{\delta}=\min \left\{\epsilon_{1} \delta, \epsilon_{2}\right\}
$$

for all $n \in \mathbb{N}$ with $n \geq n_{0}$.

Now, suppose $x, y \in \mathbb{R}^{2}$ are arbitrary, and let $S$ be the line segment joining $x$ and $y$. Then, $S$ can be broken up into $N \in \mathbb{N}$ line segments of diameter $<\delta$, where $N \leq|x-y| / \delta+1$. We can apply the previous considerations for each of these smaller (parametrized) line segments in the role of $\beta$. By what we have seen, for $n \geq n_{0}$, each of these smaller line segments has an image under $A^{-n}$ of diameter $<\widetilde{\delta}$ by (3.7). Since the concatenation of these $N$ image paths is the path $A^{-n}(S)$ connecting $A^{-n}(x)$ and $A^{-n}(y)$, we conclude that:

$$
\begin{aligned}
\left|A^{-n}(x)-A^{-n}(y)\right| & \leq \operatorname{diam}\left(A^{-n}(S)\right) \leq N \tilde{\delta} \\
& \leq(|x-y| / \delta+1) \min \left\{\epsilon_{1} \delta, \epsilon_{2}\right\} \leq \epsilon_{1}|x-y|+\epsilon_{2}
\end{aligned}
$$

for $n \geq n_{0}$, as desired.

Recall that an $\mathbb{R}$-linear map $L: \mathbb{R}^{2} \rightarrow \mathbb{R}^{2}$ is called expanding if $|\lambda|>1$ for each of the (possibly complex) roots $\lambda$ of the characteristic polynomial $P_{L}(z)=$ $\operatorname{det}\left(L-z \operatorname{id}_{\mathbb{R}^{2}}\right)$ of $L$.

Corollary 3.3 Suppose the linear map $L: \mathbb{R}^{2} \rightarrow \mathbb{R}^{2}$ is as in (3.2). Then, L is expanding.

Proof We argue by contradiction and assume that $L$ is not expanding. Choosing $\epsilon_{1}=$ $\epsilon_{2}=1 / 2$ in Lemma 3.2, we can find a number $n \in \mathbb{N}$, such that:

$$
\left|A^{n}(u)-A^{n}(v)\right| \geq 2|u-v|-1
$$

for all $u, v \in \mathbb{R}^{2}$. In other words, $A^{n}$ expands large distances roughly by the factor 2 .

Let $\lambda_{1}, \lambda_{2} \in \mathbb{C}$ be the two (possibly identical) roots of the characteristic polynomial $P(z)=\operatorname{det}\left(L-z \operatorname{id}_{\mathbb{R}^{2}}\right)$ of $L$. We may assume that $\left|\lambda_{1}\right| \leq\left|\lambda_{2}\right|$. Since $P$ has real coefficients, we have $\lambda_{2}=\overline{\lambda_{1}}$ if $\lambda_{1}$ is not real. Moreover, $\lambda_{1} \lambda_{2}=\operatorname{det}(L)=\operatorname{deg}(\bar{A})=$ $\operatorname{deg}(f) \geq 2$ (see Lemma 2.13 (iii) and Lemma 2.7 (i)). Therefore, the only possibility that $L$ can fail to be expanding is if $\lambda_{1}$ is real and $\left|\lambda_{1}\right| \leq 1$. Then, there exists $e \in \mathbb{R}^{2}$, $e \neq 0$, such that $L(e)=\lambda_{1} e$.

Let $\ell=\{t e: t \in \mathbb{R}\}$ be the line spanned by $e$. If $u, v \in \ell$ are arbitrary, then:

$$
\left|L^{n}(u)-L^{n}(v)\right|=\left|\lambda_{1}\right|^{n}|u-v| \leq|u-v| .
$$


If $\Gamma$ is the lattice chosen as in the beginning of this section, then (3.3) shows that:

$$
\begin{aligned}
A^{n}(x+\gamma)-L^{n}(x+\gamma) & =\left(A^{n} \circ \tau_{\gamma}\right)(x)-L^{n}(x)-L^{n}(\gamma) \\
& =\left(\tau_{L^{n}(\gamma)} \circ A^{n}\right)(x)-L^{n}(x)-L^{n}(\gamma) \\
& =A^{n}(x)+L^{n}(\gamma)-L^{n}(x)-L^{n}(\gamma) \\
& =A^{n}(x)-L^{n}(x)
\end{aligned}
$$

for all $x \in \mathbb{R}^{2}$. Since the lattice translations $\tau_{\gamma}, \gamma \in \Gamma$, act cocompactly on $\mathbb{R}^{2}$, this implies that there exists a constant $C \geq 0$, such that:

$$
\left|A^{n}(u)-L^{n}(u)\right| \leq C
$$

for all $u \in \mathbb{R}^{2}$. Combining this with (3.9), we see that:

$$
\left|A^{n}(u)-A^{n}(v)\right| \leq\left|L^{n}(u)-L^{n}(v)\right|+2 C \leq|u-v|+2 C
$$

for all $u, v \in \ell$. Therefore, the map $A^{n}$ expands distances along $\ell$ by at most an additive term. This is irreconcilable with (3.8), and we get a contradiction. The statement follows.

We record the following consequence.

Corollary 3.4 Suppose that the map A as in (3.1) has a fixed point $x \in \mathbb{R}^{2}$. Then, if $U$ is any open neighborhood of $x$, we have:

$$
\bigcup_{n \in \mathbb{N}_{0}} A^{n}(U)=\mathbb{R}^{2} .
$$

Moreover, if $U$ is bounded in addition, then $U \subset A^{n}(U)$ for all sufficiently large $n \in \mathbb{N}$.

Proof Let $U$ be a neighborhood of $x$. Then, there exists $\epsilon>0$, such that $B:=$ $\left\{z \in \mathbb{R}^{2}:|z-x|<\epsilon\right\} \subset U$. If $y \in \mathbb{R}^{2}$ is arbitrary, then Lemma 3.2 implies that $\left|A^{-n}(y)-x\right|=\left|A^{-n}(y)-A^{-n}(x)\right|$ is arbitrarily small for $n \in \mathbb{N}$ sufficiently large. Hence, there exist $n \in \mathbb{N}$, such that $A^{-n}(y) \in B \subset U$, and so, $y \in A^{n}(U)$. It follows that $\mathbb{R}^{2}=\bigcup_{n \in \mathbb{N}_{0}} A^{n}(U)$.

If $U$ is bounded, then there exists $R>0$, such that $U \subset B^{\prime}:=\left\{z \in \mathbb{R}^{2}:|z-x|<\right.$ $R\}$. Applying Lemma 3.2 for $\epsilon_{1}=\epsilon /(2 R)$ and $\epsilon_{2}=\epsilon / 2$, we see that:

$$
A^{-n}(U) \subset A^{-n}\left(B^{\prime}\right) \subset B \subset U
$$

for all sufficiently large $n \in \mathbb{N}$. Hence, $U \subset A^{n}(U)$ for all large $n$. 


\subsection{Transitive Action on Fibers}

Next, we will show that the group $G$ of deck transformations of the map $\Theta$ as in (3.1) acts transitively on each fiber $\Theta^{-1}(p), p \in S^{2}$. We first show that this is true in a special case.

Lemma 3.5 Let $p \in S^{2} \backslash f^{-1}(\operatorname{post}(f)), x, y \in \Theta^{-1}(p)$, and define $\bar{x}=\pi(x), \bar{y}=$ $\pi(y) \in T^{2}$. Suppose $\bar{x}$ is a fixed point of $\bar{A}$. If $\bar{y}$ is also a fixed point of $\bar{A}$ or if $\bar{x}=\bar{A}(\bar{y})$, then there exists $g \in G$, such that $g(x)=y$.

Proof Let the points $p \in S^{2}, x, y \in \mathbb{R}^{2}, \bar{x}, \bar{y} \in T^{2}$ be given as in the statement. In particular, we assume that $\bar{A}(\bar{x})=\bar{x}$. Note that:

$$
\pi(A(x))=(\pi \circ A)(x)=(\bar{A} \circ \pi)(x)=\bar{A}(\bar{x})=\bar{x},
$$

and so $x, A(x) \in \pi^{-1}(\bar{x})$. This means that $\gamma_{0}:=x-A(x) \in \Gamma$. Define $A_{0}(u)=$ $A(u)+\gamma_{0}=\left(\tau_{\gamma_{0}} \circ A\right)(u), u \in \mathbb{R}^{2}$. Note that then $A_{0}(x)=A(x)+\gamma_{0}=x$, and so $A_{0}$ has the fixed point $x$. Recall from the discussion following (3.1) that we may replace $A$ in this diagram with $A_{0}$ (while all the other maps remain the same). In other words, we are reduced to the case when $A(x)=x$ in addition to our other hypotheses.

We now consider the cases $\bar{A}(\bar{y})=\bar{y}$ and $\bar{A}(\bar{y})=\bar{x}$ separately.

Case I: $\bar{A}(\bar{y})=\bar{x}$.

This is the easy case. Note that:

$$
\pi(A(y))=(\pi \circ A)(y)=(\bar{A} \circ \pi)(y)=\bar{A}(\bar{y})=\bar{x},
$$

and so, $A(x), A(y) \in \pi^{-1}(\bar{x})$. This implies that we can find $\gamma \in \Gamma$ with $A(y)-A(x)=$ $\gamma$. Then:

$$
y=A^{-1}(A(x)+\gamma) .
$$

Thus, $g:=A^{-1} \circ \tau_{\gamma} \circ A$ is a homeomorphism on $\mathbb{R}^{2}$ with $g(x)=y$.

We want to show that $g \in G$, meaning that we need to verify that $\Theta \circ g=\Theta$. We know that $\tau_{\gamma} \in G$. Using $f \circ \Theta=\Theta \circ A$ from (3.1), we obtain:

$$
\begin{aligned}
f \circ \Theta \circ g & =\Theta \circ A \circ g=\Theta \circ \tau_{\gamma} \circ A \\
& =\Theta \circ A=f \circ \Theta .
\end{aligned}
$$

We now apply Lemma 2.14 for the branched covering maps $\Theta$ and $\Theta \circ g$. Note that $(\Theta \circ g)(x)=\Theta(y)=\Theta(x)=p$ and $p \in S^{2} \backslash f^{-1}(\operatorname{post}(f))$. It follows that $\Theta=\Theta \circ g$. We proved the statement in Case I.

Case II: $\bar{x}$ and $\bar{y}$ are fixed points of $\bar{A}$.

This case is much harder, since there is no translation $\tau_{\gamma}$ with $\gamma \in \Gamma$ that maps $A(x)$ to $A(y)$. To construct a deck transformation of $\Theta$ as in the statement, we first show that we can obtain a local one. 
Claim 1 There is a homeomorphism $\tilde{g}: U \rightarrow V$ between bounded and connected open neighborhoods $U$ and $V$ of $x$ and $y$, respectively, with $\Theta \circ \tilde{g}=\Theta$ on $U$.

To prove this, we note that our assumption $p \in S^{2} \backslash f^{-1}(\operatorname{post}(f)) \subset S^{2} \backslash \operatorname{post}(f)$ implies that $\bar{\Theta}$, and hence, also $\Theta$ has no critical point over $p$, because $\operatorname{post}(f)=$ $\bar{\Theta}(\operatorname{crit}(\bar{\Theta}))$ (see Lemma 2.7 (ii)). In particular, $\Theta$ is a local homeomorphism near both points $x, y \in \Theta^{-1}(p)$. This implies that there exist bounded and connected open neighborhoods $U \subset \mathbb{R}^{2}$ of $x, V \subset \mathbb{R}^{2}$ of $y$, and $W \subset S^{2}$ of $p$, such that $\Theta \mid U: U \rightarrow W$ and $\Theta \mid V: V \rightarrow W$ are homeomorphisms. Defining $\tilde{g}:=(\Theta \mid V)^{-1} \circ(\Theta \mid U)$ on $U$ gives the desired map, proving Claim 1.

Now, the idea is to extend $\tilde{g}$ to a deck transformation on $\mathbb{R}^{2}$ using the dynamics of $A$ near its fixed point $x$. By Corollary 3.4, we know that $U \subset A^{n}(U)$ for all sufficiently large $n \in \mathbb{N}$. Replacing $A$ with such an iterate $A^{n}$ (and, consequently, $f$ with $f^{n}$, and $\bar{A}$ with $\bar{A}^{n}$ ), we may assume that $U \subset A(U)$. Note, we then still have $\bar{A}(\bar{x})=\bar{x}$ and $\bar{A}(\bar{y})=\bar{y}$ for the new map $\bar{A}$. We make the assumption $U \subset A(U)$ from now on.

We know that $\bar{A}(\bar{y})=\bar{y}$, but, in general, the point $y$ will not be a fixed point of $A$. We have:

$$
\pi(A(y))=(\pi \circ A)(y)=(\bar{A} \circ \pi)(y)=\bar{A}(\bar{y})=\bar{y},
$$

and so, $y, A(y) \in \pi^{-1}(\bar{y})$. It follows that $A(y)=y+\gamma$ for some $\gamma \in \Gamma$. Therefore, if we define $\tilde{A}(u)=A(u)-\gamma=\left(\tau_{-\gamma} \circ A\right)(u)$ for $u \in \mathbb{R}^{2}$, then $\tilde{A}(y)=y$.

Define $U^{n}=A^{n}(U)$ for $n \in \mathbb{N}_{0}$. The sets $U^{n}$ are connected open sets containing $x$. We have $U^{n} \subset U^{n+1}$ for $n \in \mathbb{N}_{0}$, and $\bigcup_{n \in \mathbb{N}_{0}} U^{n}=\mathbb{R}^{2}$. The last fact follows from Corollary 3.4.

We now define homeomorphisms $g_{n}$ mapping $U^{n}$ into $\mathbb{R}^{2}$ recursively, by setting $g_{0}:=\tilde{g}$ on $U^{0}=U$, and

$$
g_{n+1}:=\tilde{A} \circ g_{n} \circ A^{-1} \text { on } U^{n+1}
$$

for $n \in \mathbb{N}_{0}$. Note that this makes sense, because $A^{-1}\left(U^{n+1}\right)=U^{n}$. This definition implies that:

$$
\tau_{\gamma} \circ g_{n+1} \circ A=A \circ g_{n} \text { on } U^{n}
$$

for all $n \in \mathbb{N}_{0}$.

One verifies by induction that $\Theta \circ g_{n}=\Theta$ on $U^{n}$ for all $n \in \mathbb{N}$. Indeed, this is true for $n=0$ by definition of $g_{0}=\tilde{g}$. If it is true for $n \in \mathbb{N}_{0}$, then it is also true for $n+1$, because:

$$
\begin{aligned}
\Theta \circ g_{n+1} & =\Theta \circ \tau_{-\gamma} \circ A \circ g_{n} \circ A^{-1} \\
& =\Theta \circ A \circ g_{n} \circ A^{-1} \\
& =f \circ \Theta \circ g_{n} \circ A^{-1}=f \circ \Theta \circ A^{-1} \\
& =\Theta \circ A \circ A^{-1}=\Theta .
\end{aligned}
$$


By induction, one also shows that $g_{n}(x)=y$ for all $n \in \mathbb{N}_{0}$. Indeed, $g_{0}(x)=\tilde{g}(x)=y$, and if this is true for $n \in \mathbb{N}_{0}$, then it is also true for $n+1$, because:

$$
\begin{aligned}
g_{n+1}(x) & =\left(\tilde{A} \circ g_{n} \circ A^{-1}\right)(x) \\
& =\left(\tilde{A} \circ g_{n}\right)(x)=\tilde{A}(y)=y .
\end{aligned}
$$

Claim 2 We have $g_{n+1} \mid U^{n}=g_{n}$ for all $n \in \mathbb{N}_{0}$.

To see this, we want to apply Lemma 2.14 to the branched covering map $\Theta: \mathbb{R}^{2} \rightarrow$ $S^{2}$, and the maps $g_{n}$ and $g_{n+1} \mid U_{n}$. We know that:

$$
\Theta \circ g_{n}=\Theta=\Theta \circ\left(g_{n+1} \mid U_{n}\right)
$$

on the connected open set $U_{n}$. Moreover, $g_{n}(x)=y=g_{n+1}(x)$ and the point $y$ which lies in the fiber over $p=\Theta(y)$ not containing any critical point of $\Theta$. Claim 2 follows.

Therefore, each homeomorphism $g_{n}$ extends the previous one. Since the sets $U^{n}$, $n \in \mathbb{N}_{0}$, exhaust $\mathbb{R}^{2}$, there exists a unique map $g: \mathbb{R}^{2} \rightarrow \mathbb{R}^{2}$, such that $g \mid U^{n}=g_{n}$ for all $n \in \mathbb{N}_{0}$. It is clear that $g$ is continuous and injective, because the maps $g_{n}$ have these properties. Moreover, it is clear that $g(x)=y$ and $\Theta=\Theta \circ g$ on $\mathbb{R}^{2}$.

To finish the proof in the Case II at hand, it remains to show that $g: \mathbb{R}^{2} \rightarrow \mathbb{R}^{2}$ is surjective. To do this, let us shift our attention to the images $g\left(U^{n}\right)$.

Claim 3 We have $g\left(U^{n}\right)=g_{n}\left(U^{n}\right)=\tilde{A}^{n}(V)$ for all $n \in \mathbb{N}_{0}$.

Recall that $V=\tilde{g}(U)$ was the neighborhood of $y$ defined in Claim 1. Thus, Claim 3 is true for $n=0$, since

$$
g\left(U^{0}\right)=g_{0}\left(U^{0}\right)=\tilde{g}(U)=V=\tilde{A}^{0}(V)
$$

Moreover, if it is true for $n \in \mathbb{N}_{0}$, then it is also true for $n+1$, because:

$$
\begin{aligned}
g\left(U^{n+1}\right) & =g_{n+1}\left(U^{n+1}\right)=g_{n+1}\left(A\left(U^{n}\right)\right) \\
& =\left(\tilde{A} \circ g_{n} \circ A^{-1}\right)\left(A\left(U^{n}\right)\right)=\left(\tilde{A} \circ g_{n}\right)\left(U^{n}\right) \\
& =\tilde{A}\left(\tilde{A}^{n}(V)\right)=\tilde{A}^{n+1}(V),
\end{aligned}
$$

proving Claim 3.

Recall that in (3.1), we can replace $A$ with $\tilde{A}=\tau_{-\gamma} \circ A$. Since the map $\tilde{A}$ has the fixed point $y$, we can apply Corollary 3.4 to $\tilde{A}$; so the images of the neighborhood $V$ of $y$ under iterates of $\tilde{A}$ will exhaust $\mathbb{R}^{2}$, i.e., we have $\bigcup_{n \in \mathbb{N}} \tilde{A}^{n}(V)=\mathbb{R}^{2}$. Using Claim 3, we conclude that $g$ is surjective. This finishes the proof of Case II. The statement follows.

We now show transitivity of the action of $G$ on the fibers of $\Theta$.

Lemma 3.6 Let $p \in S^{2}$ and $x, y \in \Theta^{-1}(p)$. Then, there exists $g \in G$, such that $g(x)=y$. 
Proof We will first show the existence of a point $p_{0} \in S^{2} \backslash \operatorname{post}(f)$ for which $G$ acts transitively on the fiber $\Theta^{-1}\left(p_{0}\right)$, and then deal with the general case.

The periodic points of $f$ are dense in $S^{2}$ (see [1, Corollary 9.2]); in particular, we can find a periodic point $p_{0} \in S^{2} \backslash \operatorname{post}(f)$. By replacing $f$ with suitable iterates $f^{n}$ (and $\bar{A}$ with $\bar{A}^{n}$ ), we may assume that $p_{0}$ is a fixed point of $f$. Note that then we still have $p_{0} \in S^{2} \backslash \operatorname{post}(f)$, because the postcritical sets of a Thurston map and any of its iterates agree.

Since $p_{0}$ is a fixed point of $f$, the map $\bar{A}$ sends the set $\bar{\Theta}^{-1}\left(p_{0}\right)$ into itself. It follows that each point in $\bar{\Theta}^{-1}\left(p_{0}\right)$ is either a periodic point of $\bar{A}$ or is mapped to a periodic point of $\bar{A}$ under all sufficiently high iterates of $\bar{A}$. If we again replace $f$ and $\bar{A}$ with carefully chosen iterates, we can reduce ourselves to the following situation: $p_{0} \in S^{2} \backslash \operatorname{post}(f)$ is a fixed point of $f$, the set $\bar{\Theta}^{-1}\left(p_{0}\right)$ contains at least one fixed point of $\bar{A}$, and each point in $\bar{\Theta}^{-1}\left(p_{0}\right)$ is either a fixed point of $\bar{A}$ or mapped to a fixed point by $\bar{A}$. Moreover, since $p_{0}$ is a fixed point of $f$, and $p_{0} \in S^{2} \backslash \operatorname{post}(f)$, we have $p_{0} \in S^{2} \backslash f^{-1}(\operatorname{post}(f))$. We pick a fixed point $\bar{x} \in \bar{\Theta}^{-1}\left(p_{0}\right)$ of $\bar{A}$, and a point $x \in \mathbb{R}^{2}$ with $\pi(x)=\bar{x}$. Then, $x \in \Theta^{-1}\left(p_{0}\right)$.

Now, let $y \in \Theta^{-1}\left(p_{0}\right)$ be arbitrary. Then, $\bar{y}:=\pi(y) \in \bar{\Theta}^{-1}\left(p_{0}\right)$. By our choices, $\bar{y}$ is a fixed point of $\bar{A}$ or $\bar{z}:=\bar{A}(\bar{y})$ is a fixed point of $\bar{A}$. In the first case, there exists $g \in G$, such that $g(x)=y$ by the first part of Lemma 3.5.

In the second case, $\bar{z}$ is a fixed point of $\bar{A}$. Pick $z \in \mathbb{R}^{2}$, such that $\pi(z)=\bar{z}$. Then, $z \in \bar{\Theta}^{-1}\left(p_{0}\right)$, and again, there exists $g_{1} \in G$ with $g_{1}(x)=z$. Moreover, by Lemma 3.5, there exists $g_{2} \in G$, such that $g_{2}(z)=y$. Then, $g:=g_{2} \circ g_{1} \in G$ and $g(x)=y$.

We see that $x$ can be sent to any point in $\Theta^{-1}\left(p_{0}\right)$ by a suitable element $g$ in the group $G$; it follows that $G$ acts transitively on $\Theta^{-1}\left(p_{0}\right)$.

Now, let $p \in S^{2}$ and $x, y \in \Theta^{-1}(p)$ be arbitrary. Then, we can find a path $\alpha:[0,1] \rightarrow S^{2}$ with $\alpha(0)=p$ and $\alpha(1)=p_{0}$, so that $\alpha((0,1]) \subset S^{2} \backslash \operatorname{post}(f)$. Therefore, $\alpha$ lies in $S^{2} \backslash \operatorname{post}(f)$ with the possible exception of its initial point $p$. Since $x, y \in \Theta^{-1}(p)$, we can lift the path $\alpha$ under the branched covering map $\Theta: \mathbb{R}^{2} \rightarrow S^{2}$ to paths $\beta_{1}, \beta_{2}:[0,1] \rightarrow \mathbb{R}^{2}$, such that:

$$
\Theta \circ \beta_{1}=\Theta \circ \beta_{2}=\alpha
$$

$\beta_{1}(0)=x$, and $\beta_{2}(0)=y($ see $[1$, Lemma A.18]).

Let $x^{\prime}=\beta_{1}(1)$ and $y^{\prime}=\beta_{2}(1)$. Then:

$$
\Theta\left(x^{\prime}\right)=\Theta\left(\beta_{1}(1)\right)=\alpha(1)=p_{0},
$$

and so, $x^{\prime} \in \Theta^{-1}\left(p_{0}\right)$. Similarly, $y^{\prime} \in \Theta^{-1}\left(p_{0}\right)$.

By the first part of the proof, there exists $g \in G$, such that $g\left(x^{\prime}\right)=y^{\prime}$. Let $\beta_{3}:=$ $g \circ \beta_{1}$. Then:

$$
\beta_{3}(1)=g\left(\beta_{1}(1)\right)=g\left(x^{\prime}\right)=y^{\prime}=\beta_{2}(1)
$$


and

$$
\Theta \circ \beta_{3}=\Theta \circ g \circ \beta_{1}=\Theta \circ \beta_{1}=\alpha .
$$

Therefore, $\beta_{3}$ is a lift of $\alpha$ under $\Theta$ with the same endpoint as $\beta_{2}$. Since $\Theta$ is a covering map over $S^{2} \backslash \operatorname{post}(f)$ and $\alpha \mid(0,1] \subset S^{2} \backslash \operatorname{post}(f)$, it follows that $\beta_{2}\left|(0,1]=\beta_{3}\right|(0,1]$ (see [1, Lemma A.6 (i)]). Hence, $\beta_{3}(0)=\beta_{2}(0)$ by continuity, and so:

$$
g(x)=g\left(\beta_{1}(0)\right)=\beta_{3}(0)=\beta_{2}(0)=y .
$$

This shows that $G$ acts transitively on the fiber $\Theta^{-1}(p)$.

We are now ready to prove the implication (i) $\Rightarrow$ (ii) in Theorem 1.2.

Proposition 3.7 Suppose $f: S^{2} \rightarrow S^{2}$ is an expanding Thurston map that is a quotient of a torus endomorphism. Then, $f$ has a parabolic orbifold.

Proof We can use all the previous considerations for the maps as in (3.1) and the deck transformation group $G$ of $\Theta$.

Let $p \in S^{2}$ be arbitrary, and $\bar{x}, \bar{y} \in \bar{\Theta}^{-1}(p)$. Then, there exist points $x, y \in \mathbb{R}^{2}$, such that $\pi(x)=\bar{x}$ and $\pi(y)=\bar{y}$. Then, $\Theta(x)=(\bar{\Theta} \circ \pi)(x)=\bar{\Theta}(\bar{x})=p=\Theta(y)$. By Lemma 3.6, there exists $g \in G$, such that $g(x)=y$. Since $g$ is a homeomorphism, $\pi$ is a local homeomorphism, and both maps preserve orientation, we have $\operatorname{deg}(g, x)=1$ and $\operatorname{deg}(\pi, x)=\operatorname{deg}(\pi, y)=1$. Since local degrees are multiplicative under compositions (see (2.2)), we conclude:

$$
\begin{aligned}
\operatorname{deg}(\bar{\Theta}, \bar{y}) & =\operatorname{deg}(\bar{\Theta}, \bar{y}) \cdot \operatorname{deg}(\pi, y)=\operatorname{deg}(\Theta, y) \\
& =\operatorname{deg}(\Theta, y) \cdot \operatorname{deg}(g, x)=\operatorname{deg}(\Theta \circ g, x) \\
& =\operatorname{deg}(\Theta, x)=\operatorname{deg}(\bar{\Theta}, \bar{x}) .
\end{aligned}
$$

Therefore, the local degree of $\bar{\Theta}$ in each fiber $\bar{\Theta}^{-1}(p), p \in S^{2}$, is constant. Now, Lemma 2.7 (iii) implies that $f$ has a parabolic orbifold.

\section{From Parabolic Orbifolds to Lattès-Type Maps}

In this section, we will prove the implication (ii) $\Rightarrow$ (iii) in Theorem 1.2. We first establish an auxiliary fact that helps us in identifying Lattès-type maps.

Lemma 4.1 Let $f: S^{2} \rightarrow S^{2}$ be a map that is topologically conjugate to a Lattès-type map. Then, $f$ itself is Lattès-type map.

As we will see, the proof is fairly straightforward. There is a subtlety though that arises from the fact that, by definition, a branched covering map is orientationpreserving (see (2.1)), but a homeomorphism as in the definition of topological conjugacy may actually reverse orientation. To address this, we have to compensate by complex conjugation on $\mathbb{C}$ in a suitable way. 
In the following, we denote by $\sigma: \mathbb{C} \rightarrow \mathbb{C}, \sigma(z)=\bar{z}$ for $z \in \mathbb{C}$, complex conjugation on $\mathbb{C}$. Note that this is an $\mathbb{R}$-linear orientation-reversing isometry on $\mathbb{C}$ with $\sigma^{-1}=\sigma$.

Proof of Lemma 4.1 Suppose $f: S^{2} \rightarrow S^{2}$ is a map that is topological conjugate to a Lattès-type map $\widehat{f}: \widehat{S}^{2} \rightarrow \widehat{S}^{2}$. Here, $\widehat{S}^{2}$ is another topologically 2-sphere. Both $S^{2}$ and $\widehat{S}^{2}$ carry some fixed orientations.

According to Definition 2.8 , there exists a crystallographic group $\widehat{G}$ acting on $\mathbb{C} \cong \mathbb{R}^{2}$, an $\widehat{G}$-equivariant (real) affine map $\widehat{A}: \mathbb{C} \rightarrow \mathbb{C}$, and a branched covering map $\widehat{\Theta}: \mathbb{C} \rightarrow \widehat{S}^{2}$ induced by $\widehat{G}$ with $\widehat{f} \circ \widehat{\Theta}=\widehat{\Theta} \circ \widehat{A}$. Moreover, the linear part $L_{\widehat{A}}$ of $\widehat{A}$ satisfies $\operatorname{det}\left(L_{\widehat{A}}\right)>1$.

Since $f$ and $\widehat{f}$ are topologically conjugate, there exists a homeomorphism $h: \widehat{S}^{2} \rightarrow$ $S^{2}$, such that $f \circ h=h \circ \widehat{f}$. We now have to distinguish two cases according to whether the homeomorphism $h: \widehat{S}^{2} \rightarrow S^{2}$ preserves or reverses orientation. We will treat the latter, slightly more difficult case in detail, and then comment on the small modifications for the former case.

Therefore, we now assume that $h: \widehat{S}^{2} \rightarrow S^{2}$ is orientation-reversing. We define $\Theta:=h \circ \widehat{\Theta} \circ \sigma$. Then, $\Theta: \mathbb{C} \rightarrow S^{2}$ is a branched covering map, as easily follows from the definitions and the fact that $\widehat{\Theta}: \mathbb{C} \rightarrow \widehat{S}^{2}$ is a branched covering map. Here, it is important that in the definition of $\Theta$, we compensate postcomposition of $\widehat{\Theta}$ with the orientation-reversing homeomorphism $h$ by precomposition with the orientationreversing homeomorphism $\sigma$ to make $\Theta$ orientation-preserving.

We conjugate everything else by $\sigma$. More precisely, we define $G:=\{\sigma \circ g \circ \sigma$ : $g \in \widehat{G}\}$. It is clear that $G$ is a crystallographic group on $\mathbb{C}$ and $\Theta$ is induced by $G$. Moreover, we let $A:=\sigma \circ \widehat{A} \circ \sigma$. Then, $A$ is a (real) affine map on $\mathbb{C} \cong \mathbb{R}^{2}$ that is $G$-equivariant. For the linear part $L_{A}$ of $A$, we have $L_{A}=\sigma \circ L_{\widehat{A}} \circ \sigma$, and so, $\operatorname{det}\left(L_{A}\right)=\operatorname{det}\left(L_{\widehat{A}}\right)>1$.

We can summarize the relations of all the maps considered in the following diagram, which is obviously commutative:

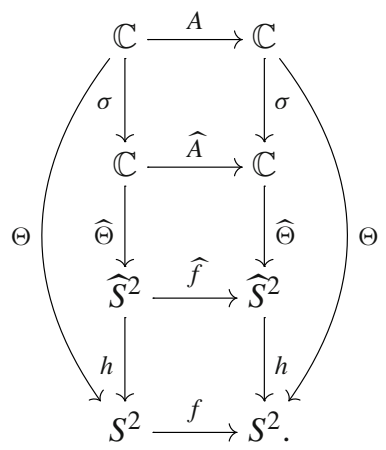

It follows that $f$ is a Lattès-type map.

If $h$ is orientation-preserving, the map $\sigma$ is not needed in the previous argument. Formally, we can just replace $\sigma$ with the identity map on $\mathbb{C}$. Therefore, $f$ is also a Lattès-type map in this case. The statement follows. 
After this preparation, we now prove the implication (ii) $\Rightarrow$ (iii) in Theorem 1.2.

Proposition 4.2 Let $f: S^{2} \rightarrow S^{2}$ be an expanding Thurston map with a parabolic orbifold. Then, $f$ is a Lattès-type map.

Proof Let $f$ be as in the statement, and $\alpha=\alpha_{f}$ be the ramification function of $f$. Since $f$ has a parabolic orbifold $\mathcal{O}_{f}=(\widehat{\mathbb{C}}, \alpha)$, the signature of $\mathcal{O}_{f}$ is in the list (see (2.4)):

$$
(\infty, \infty),(2,2, \infty),(2,4,4),(2,3,6),(3,3,3),(2,2,2,2)
$$

The fact that $f$, or equivalently $f^{2}$, is expanding (see [1, Lemma 6.5]), rules out the signatures $(\infty, \infty)$ and $(2,2, \infty)$. Indeed, if a Thurston map $f$ has one of these signatures, then $f$ or $f^{2}$ is a Thurston polynomial (i.e., there is a point that is completely invariant under the map); see the discussion after [1, Lemma 7.4]. However, no Thurston polynomial is expanding (see [1, Lemma 6.8]). This means that $\alpha$ does not attain the value $\infty$; so $f$ has no periodic critical points (see [1, Proposition 2.2 (ii)]) and the signature of $\mathcal{O}_{f}$ is among:

$$
(2,4,4),(2,3,6),(3,3,3),(2,2,2,2) \text {. }
$$

In the first three cases, $f$ has precisely three postcritical points, and is, hence, Thurston equivalent to a rational Thurston map $R: \widehat{\mathbb{C}} \rightarrow \widehat{\mathbb{C}}$ (see $[1$, Theorem 7.2 and Lemma 2.5]). Since $f$ and $R$ are Thurston equivalent, the orbifolds of $f$ and $R$ have the same signatures (see [1, Proposition 2.15]), namely $(2,4,4),(2,3,6)$, or $(3,3,3)$. In particular, $R$ has a parabolic orbifold.

Moreover, the ramification function of $R$ only takes finite values which again implies that $R$ has no periodic critical points. Hence, $R$ is expanding (see [1, Proposition 2.3]) and actually a Lattès map (see Theorem 2.5). Since $f$ and $R$ are Thurston equivalent, and both Thurston maps are expanding, it follows that $f$ and $R$ are topologically conjugate (see Theorem 2.4). Now, Lemma 4.1 implies that $f$ is a Lattès-type map (note that the Lattès map $R$ is also of Lattès-type).

It remains to consider the case where the signature of $\mathcal{O}_{f}$ is equal to $(2,2,2,2)$. We know that $f$ is Thurston equivalent to a Lattès-type map $\widehat{f}: \widehat{S}^{2} \rightarrow \widehat{S}^{2}$ (see Proposition 2.10). The proof of Proposition 2.10 (see [1, pp. 74-77]) shows that $f$ is a quotient of a torus endomorphism and that the affine map $\widehat{A}$ for $\widehat{f}$ as in Definition 2.8 can be chosen, so that its linear part $L_{\widehat{A}}$ is equal to the map $L$ as in (3.2). It follows that $L_{\widehat{A}}=L$ is expanding as a linear map (see Lemma 3.3). This, in turn, implies that $\widehat{f}$ is expanding as a Thurston map (see Proposition 2.12). Now, we can again conclude that $f$ and $\widehat{f}$ are topologically conjugate, and hence $f$ is a Lattès-type map by Lemma 4.1 .

We can now wrap up the proof of Theorem 1.2.

Proof of Theorem 1.2 The implications (i) $\Rightarrow$ (ii) and (ii) $\Rightarrow$ (iii) were proved in Propositions 3.7 and 4.2, respectively. The implication (iii) $\Rightarrow$ (i) was explicitly stated in Proposition 2.9. 
Open Access This article is licensed under a Creative Commons Attribution 4.0 International License, which permits use, sharing, adaptation, distribution and reproduction in any medium or format, as long as you give appropriate credit to the original author(s) and the source, provide a link to the Creative Commons licence, and indicate if changes were made. The images or other third party material in this article are included in the article's Creative Commons licence, unless indicated otherwise in a credit line to the material. If material is not included in the article's Creative Commons licence and your intended use is not permitted by statutory regulation or exceeds the permitted use, you will need to obtain permission directly from the copyright holder. To view a copy of this licence, visit http://creativecommons.org/licenses/by/4.0/.

\section{References}

1. Bonk, M., Meyer, D.: Expanding Thurston Maps. American Mathematical Society, Providence (2017)

2. Cannon, J.W., Floyd, W.J., Parry, W.R., Pilgrim, K.M.: Nearly Euclidean Thurston maps. Conform. Geom. Dyn. 16, 209-255 (2012)

3. Douady, A., Hubbard, J.H.: A proof of Thurston's topological characterization of rational functions. Acta Math. 171, 263-297 (1993)

4. Milnor, J.: On Lattès maps. In: Dynamics on the Riemann Sphere. Eur. Math. Soc., Zürich, pp. 9-43 (2006)

Publisher's Note Springer Nature remains neutral with regard to jurisdictional claims in published maps and institutional affiliations. 